\title{
FATORES RELACIONADOS A INTERNACIONALIZAÇÃO DAS REDES DE FRANQUIAS BRASILEIRAS: DISPERSÃO GEOGRÁFICA E PERTENCIMENTO A GRUPOS
}

\author{
Vanessa Pilla Bretas ${ }^{1}$, Jefferson Ricardo Galetti, Thelma Valéria Rocha \\ Escola Superior de Propaganda e Marketing - ESPM, São Paulo, (Brasil) \\ Universidade Federal do Rio de Janeiro, Rio de Janeiro, (Brasil)
}

DETALHES DO ARTIGO
Artigo Convidado:
Recebido: 16 de agosto de 2018
Aceito: 23 de dezembro de 2018
Disponível online: 01 de janeiro de 2019
Editor Científico
Ilan Avrichir

\section{Palavras-chaves:}

Franchising Internacional

Franquias

Internacionalização

Dispersão Geográfica

\begin{abstract}
RESUMO
Este artigo investiga os fatores que explicam o baixo índice de internacionalização das redes de franquias brasileiras. O objetivo é identificar se por um lado os recursos da empresa, como experiência de monitoramento, dispersão geográfica no mercado doméstico e reputação, e, por outro, aspectos institucionais, como pertencimento a grupos empresariais, influenciam a probabilidade das redes de franquias brasileiras se internacionalizarem. Utilizando dados secundários disponibilizados pela Associação Brasileira de Franchising (ABF), são investigadas as relações entre quatro aspectos influenciadores $e$ as chances de internacionalização por meio de uma regressão logística para uma base de 420 redes de franquias brasileiras, sendo 49 dessas internacionalizadas e 371 não internacionalizadas. Os resultados confirmaram as hipóteses relacionadas à dispersão geográfica nacional, franqueadores com maior dispersão geográfica no mercado doméstico estão mais propensos a se internacionalizarem, e pertencimento a grupos, franqueadores que pertencem a grupos estão mais propensos a se internacionalizarem. Como contribuição, este estudo inova ao comprovar quantitativamente que a dispersão geográfica nacional, e pertencer a grupos são fatores que determinam a internacionalização de redes franquia de mercados emergentes, como o Brasil.
\end{abstract}

C 2018 Internext | ESPM. Todos os direitos reservados!

\section{INTRODUÇÃO}

O setor de franchising adquiriu grande relevância no mundo e principalmente no Brasil na última década. De acordo com o WFC - World Franchise Council, em ranking elaborado pela ABF em 2016, o Brasil ocupou o quarto lugar no ranking mundial em número de redes de franquia, com 3.039 marcas, e o sexto lugar no ranking de número de unidades, com 142.593 unidades. O faturamento do setor de franquias no Brasil cresceu 40,9\% em cinco anos, de 2012 a 2016 (ABF, 2016).

No entanto, a presença internacional das redes brasileiras de franquia ainda é modesta. Em pesquisa realizada pela Associação Brasileira de Franchising (ABF) em 2016, apenas 4,5\% das redes franqueadoras brasileiras possuíam operações no exterior (consideram-se unidades próprias, franqueadas e máster franqueados). Além disso, menos que $1 \%$ do faturamento de $43 \%$ das empresas que possuem unidades próprias e/ou franqueadas no exterior é proveniente dessas operações internacionais (ABF, 2015).

O índice de internacionalização de redes de franquias brasileiros é baixo, principalmente quando comparado ao índice dos Estados Unidos, mercado maduro no setor de franchising. Cerca de 800 redes de franquias do país, ou $20 \%$ das 3.828 redes estadounidenses (2016), operam internacionalmente de acordo com a IFA - International Franchise Association.

C 2018 Internext | ESPM. Todos os direitos reservados! DOI: 10.18568/internext.v14i1.495

\footnotetext{
${ }^{1}$ Contato do autor E-mail: vpbretas@gmail.com
} 
O índice de internacionalização da franchising brasileira permanece estável na casa dos $5 \%$ há pelo menos 6 anos (Rocha et al., 2016), representando 142 redes (2017). Os Estados Unidos registram crescimentos anuais acima de 5\% no índice desde 1985. Mesmo sendo o maior mercado em número de unidades e o terceiro maior em redes (ABF / World Franchise Council, 2016), com melhor desempenho relativo de internacionalização em relação ao Brasil, estudos para entender os motivos pelos quais a maioria das redes de franquia dos Estados Unidos ainda não operam internacionalmente são desenvolvidos desde a década de 1990 (Aydin \& Kacker, 1990; Kedia et al., 1994).

Neste sentido, este estudo visa entender, a partir da visão baseada em recursos e da visão baseada em instituições, quais fatores contribuem para este baixo índice de internacionalização de redes de franquias brasileiras.

A internacionalização das operações é uma escolha estratégica e, portanto, é resultante da interação da empresa, no caso deste estudo, da rede franqueadora, com a indústria a qual pertence, aos recursos e capacidades disponíveis e com o ambiente institucional (Porter, 1980; Barney, 1991; Peng, 2002).

A visão baseada na indústria (Porter, 1980; 1985), discute as condições e a extensão em que a indústria determina a estratégia e desempenho das firmas. Já a visão baseada em recursos, segundo Barney (1991), postula a existência de diferenças específicas nos recursos das firmas que direcionam a estratégia e desempenho organizacional. A visão baseada em instituições, que completa o tripé téorico da estratégia, considera que as instituições são relevantes para a estratégia e desempenho (Peng, 2002).

Abordagens multiteóricas podem ser utilizadas para analisar escolhas estratégicas complexas, como é o caso da decisão pela internacionalização por empresas de mercados emergentes como o Brasil (Gaur et al., 2014). Neste sentido, o baixo índice de internacionalização das redes de franquia brasileiras pode ser avaliado sob uma perspectiva mutiteórica. Negócios internacionais e estratégia são abordagens que se aproximam e se integram (Peng et al., 2008, p. 920).

Recursos e vantagens de propriedade são importantes para internacionalização. Madanoglu et al. (2017) identificam uma série de estudos sobre franchising internacional desenvolvidos nas décadas de 1990 e 2000 que partem da visão baseada em recursos para analisar fatores que influenciam a internacionalização de redes de franquia, como o tamanho da rede, experiência no sistema de franchising, taxa de crescimento, royalties e taxas, capacidade de monitoramento e dispersão geográfica doméstica (Kedia, Ackerman, Bush \& Justis, 1994; Fladmoe-Lindquist, 1996, Shane, 1996, Elango, 2007; Perrigot, López-Fernández \& Eroglu, 2013).

As características do ambiente institucional também podem influenciar a expansão de redes de franquias para mercados internacionais (Aliouche et al., 2015). A experiência das franqueadoras de economias emergentes em seus mercados domésticos com instituições fracas pode ser um recurso valioso para operar em outros mercados similares. Muitas empresas se organizam em grupos empresariais para superar vazios institucionais.

Comb et al. (2009) afirmam que as pesquisas sobre franchising podem ser beneficiadas por uma maior diversidade teórica e a teoria institucional pode ser promissora pois as forças sociais e normativas aumentam o poder explicativo além do oferecido pelas explicações econômicas.

Os estudos sobre franchising internacional são predominantemente focados em redes de franquias de países desenvolvidos. Welsh, Alon e Falbe (2006) realizam uma revisão de literatura sobre franchising em mercados emergentes. São analisados 31 artigos, de 1988 a 2005, que tratam sobre Europa central e oriental, México e América do Sul, Ásia e outras áreas.

Todos os artigos analisados tratam sobre o potencial dos mercados emergentes, as oportunidades e riscos, vantagens e desvantagens para franqueadores ingressarem nesses mercados, porém nenhum estudo aborda o ponto de vista de franqueadores provenientes de mercados emergentes. Dant, Grünhagen e Windsperger (2014) apontam nove áreas de pesquisa em franchising a serem exploradas, entre elas o estudo do setor em países não inseridos no contexto da América do Norte.

Neste sentido, este artigo contribui do ponto de vista teórico e empírico ao utilizar uma abordagem multiteórica (visão baseada em recursos e em instituições) para analisar a questão proposta e ao 
Fatores Relacionados a Internacionalização das Redes de Franquias Brasileiras: Dispersão Geográfica e Pertencimento a Grupos

verificar fatores de influência para a internacionalização de redes provenientes de um mercado emergente.

Desta forma, com o objetivo de identificar fatores que interferem na internacionalização de redes de franquias brasileiras, são investigados quatro aspectos: capacidade de monitoramento; dispersão geográfica; reputação (mensurado pela dimensão possuir selo de excelência $A B F$ ); e pertencimento a grupos. Foi utilizada uma base de 420 redes de franquias brasileiras, das quais 49 estão internacionalizadas e 371 não estão no exterior.

A estrutura deste artigo contempla referencial teórico elucidando o franchising como modo de entrada internacional, respaldado por duas lentes teóricas - a Visão Baseada em Recursos e a Visão Baseada em Instituições. A metodologia de pesquisa, ancorada pela base teórica, utiliza a regressão logística como técnica estatística de análise. Nas seções seguintes são apresentados resultados e a discussão, e considerações finais.

\section{REFERENCIAL TEÓRICO}

Por seu dinamismo e diversidade, a área de negócios internacionais pode ser estudada sob diferentes perspectivas e correntes teóricas. Os estudos desenvolvidos até a década de 1970 apresentavam maior influência da área de economia industrial, abordando questões em nível macro como comercialização de produtos e fluxo de investimentos entre países. A partir da década de 70, os estudos na área de negócios internacionais passaram também a abordar aspectos do nível da firma, se aproximando das questões trabalhadas no campo de gestão estratégica (Peng, 2001). Combs et al. (2009) consideram que a perspectiva multiteórica é relevante para o estudo de franchising internacional.

Este estudo, procura integrar ambas as teorias Visão baseada e recursos (RBV) e Visão baseada em instituições (IBV) - para um melhor entendimento dos fatores que impactam a internacionalização das redes de franquias brasileiras.

\section{Franchising Internacional na Perspectiva de RBV}

Uma das lentes teóricas proeminentes e com influência crescente em negócios internacionais é a visão baseada em recursos (Resource-Based View RBV). O foco em questões relacionadas à firma e seu caráter de compatibilidade e diálogo com outras perspectivas teóricas possibilitaram a difusão da RBV no campo de negócios internacionais (Peng, 2001).
A visão baseada em recursos examina a ligação entre as características internas ou recursos das firmas e a implantação de estratégias que visam melhorar sua performance. Sob esta perspectiva, a firma é uma coleção de recursos historicamente determinados aos quais está atrelada de forma semipermanente (Barney, 1991).

Na perspectiva da RBV, as empresas pensam suas estratégias de internacionalização com base na exploração de capacidades geradas pelos recursos que possuem e que melhor respondem às oportunidades externas (Moraes et al., 2006). Ou seja, um aspecto fundamental para o sucesso da internacionalização é a exploração e combinação dos recursos e capacidades da firma e o desenvolvimento de novas capacidades. Os recursos e vantagens de propriedade das firmas são importantes para a internacionalização (Gaur et al., 2013; Lockett et al., 2009).

Face a uma revisão dos principais trabalhos na literatura sobre RBV em negócios internacionais, Peng (2001) identifica quatro áreas prioritárias: 1. Gestão de multinacionais, 2. Alianças estratégicas, 3. Empreendedorismo internacional e 4. Modos de entrada, e argumenta que, em referência aos modos de entrada em mercados internacionais, a visão baseada em recursos assume que a escolha pelo modo de entrada é impulsionada tanto pelos recursos e vantagens específicas das firmas quanto pelos possíveis recursos e capacidades que podem ser adquiridos nestas novas operações.

O sistema de franchising pode ser entendido como um modo de entrada em mercados estrangeiros (Cavusgil, T., Knight, G., \& Riesenberger, J., 2010). O franchising é um modo de entrada de controle parcial, uma vez que os franqueados adquirem o controle da operação em troca do pagamento de taxas e de acordo com os termos de um contrato (Osland, Taylor \& Zou, 2001). Além disso, o franchising é uma maneira de ingressar em mercados internacionais com um menor comprometimento de recursos próprios e menor risco por parte da franqueadora (Kedia et al., 1994).

Segundo Fladmoe-Lindquist (1996), a visão baseada em recursos é uma perspectiva teórica adequada ao estudo do processo de internacionalização de redes de franquias. Castrogiovanni, Combs e Justis (2006) também ressaltam a importância da visão baseada em recursos para entender o franchising internacional pois, de acordo com os autores, as firmas atingem um 
determinado nível de recursos tangíveis e intangíveis no mercado doméstico antes de expandirem internacionalmente.

Estudos anteriores (Shane, 1996; FladmoeLindquist, 1996) identificaram que os recursos e capacidades necessários para que redes de franquias operem em mercados domésticos são diferentes do que os recursos e capacidades que as possibilitam operar internacionalmente.

No sistema de franchising, a internacionalização tornou-se mais popular a partir da década de 1970 (Cavusgil et al., 2010). Geralmente, o objetivo das redes de franquias é sobreviver e aumentar seus lucros mantendo os riscos em um nível baixo. Eroglu (1992) entende que a decisão pela internacionalização depende dos riscos e benefícios percebidos. Se a percepção dos benefícios for maior que a percepção dos riscos, maior será a intenção de internacionalizar.

Fatores internos ou organizacionais e fatores externos ou ambientais determinam a percepção de riscos e benefícios. A escolha da forma de entrada e operação em mercados internacionais depende destes fatores organizacionais e ambientais (Eroglu, 1992; Alon, 2000). Fatores organizacionais correspondem à estrutura da firma e aspectos gerenciais, como seu histórico, o tamanho da firma, experiência internacional prévia, as características do tomador de decisão no que diz respeito à tolerância ao risco e percepção de vantagem competitiva em relação aos mercados internacionais.

Além destes fatores, os objetivos estratégicos de aumento de vendas e lucros, a expansão do mercado e o desejo de ser conhecida como uma empresa internacional contribuem para a tomada de decisão. Os fatores ambientais que influenciam na opção pela internacionalização consistem no aumento da competição no mercado doméstico, saturação do mercado doméstico, influência de agentes de mudança externos como potenciais franqueados interessados em investir na firma e disposição favorável percebida do ambiente externo (Alon, 2000; Doherty, 2007).

\section{Experiência de Monitoramento}

Para operar em mercados estrangeiros os franqueadores precisam ter recursos e algumas capacidades desenvolvidas como pré-requisitos. As capacidades necessárias em franchising internacional são diferentes das capacidades para operar em franchising doméstico e requerem constante aprendizado e desenvolvimento (Elango, 2007; Fladmoe-Lindquist, 1996; Shane, 1996).

Um aspecto fundamental para operar em mercados internacionais é a capacidade de monitoramento de franqueados. A habilidade de monitoramento é uma capacidade importante que distingue franqueadores domésticos de franqueadores internacionais pois 0 acompanhamento dos franqueados internacionais é mais difícil e complicado do que acompanhar franqueados domésticos. A distância geográfica, cultural e social podem ser um desafio para o monitoramento de operações internacionais (Elango, 2007; Fladmoe-Lindquist, 1996; Shane, 1996).

Os resultados de uma pesquisa desenvolvida por Shane (1996) indicam que é possível identificar franqueadores dos Estados Unidos com maior probabilidade de expandirem suas operações para o exterior analisando sua estrutura de preços e capacidade de monitoramento. Franqueadores podem monitorar as instalações do franqueado, analisar registros e estabelecer e averiguar o uso de equipamentos e normas. "Portanto, o monitoramento é uma capacidade que os franqueadores desenvolvem" (Shane, 1996). O monitoramento previne investimentos ineficientes.

As empresas que se internacionalizam por meio de franchising geralmente já adotam o modelo no mercado doméstico, o que facilita o processo por ser uma fórmula testada (Quinn \& Alexander, 2002; Doherty, 2007). As firmas que atuam como franqueadoras no mercado doméstico possuem uma estrutura organizacional voltada ao suporte e monitoramento de unidades franqueadas, o que facilita as operações internacionais (Kedia et al., 1994).

Franqueadores com maior tempo de existência e número maior de unidades franqueadas em relação às unidades próprias são mais propensos a operar internacionalmente, pois desenvolveram capacidades para monitorar seus franqueados. Estudos prévios indicam que a experiência de monitoramento do franqueador pode ser influenciada por dois fatores: proporção de unidades franqueadas em relação às próprias, e o número de anos em que a empresa atua no sistema de franchising (Huszagh et al., 1992; Elango, 2007).

Elango (2007) identificou forte relação entre experiência de monitoramento e a probabilidade do franqueador operar em mercados estrangeiros. $\mathrm{O}$ desenvolvimento desta capacidade pode ser aprimorado aumentando o tamanho do sistema de 
Fatores Relacionados a Internacionalização das Redes de Franquias Brasileiras: Dispersão Geográfica e Pertencimento a Grupos

franquia, a alocação de recursos para seu desenvolvimento e através da aprendizagem ao longo do tempo.

Neste sentido, propõe-se a seguinte hipótese para a realidade das redes de franquias brasileiras:

H1: Franqueadores com maior experiência de monitoramento de franqueados estão mais propensos a se internacionalizarem.

\section{Dispersão Geográfica no Mercado Doméstico}

A expansão internacional das redes de franquias aumenta a complexidade e seus custos de monitoramento. Os efeitos da dispersão geográfica são maiores para os franqueadores multinacionais do que para franqueadores locais ou nacionais à medida que cruzam as fronteiras nacionais (Castrogiovanni et al., 2006). A capacidade de gerenciar a distância geográfica é uma grande preocupação no contexto do franchising internacional (Fladmoe-Lindquist, 1996; Huszagh et al., 1992).

Neste sentido, a dispersão geográfica das redes de franquias no mercado doméstico também podem favorecer e desenvolver a capacidade de monitoramento e controle do franqueador em relação aos franqueados. Quanto maior a abrangência de atuação da rede no mercado doméstico, mais estruturados devem ser seus mecanismos de controle e acompanhamento. Portanto, a experiência das redes de franquia com as operações domésticas pode ser fonte de aprendizado e capacitá-las para operar em mercados internacionais.

Estudos anteriores abordaram a dispersão geográfica como fator relevante antecedente à decisão de internacionalizar as operações. Franqueadores com muitas unidades em diferentes localizações possuem melhores oportunidades para aproveitar vantagens de economia de escala tanto no que diz respeito ao monitoramento quanto em relação ao retorno e promoção, pois localizações distintas trazem diferentes níveis de retorno e de risco.

Ou seja, a internacionalização é um prolongamento ou extensão da expansão para franqueadores com maior dispersão geográfica (Huszagh et al., 1992; Alon et al. 2012; Pedro, Filipe \& Reis, 2008; Madanoglu et al., 2017).

De acordo com esta perspectiva, um maior escopo da dispersão geográfica doméstica da rede de franquia poderia indicar maior propensão à internacionalização.
H2: Franqueadores com maior dispersão geográfica no mercado doméstico estão mais propensos a se internacionalizarem

\section{Reputação}

Estudos anteriores apontaram a importância de recursos intangíveis, como reconhecimento da marca e reputação, para o sucesso de uma rede de franquias. A busca por legitimidade e reputação do mercado é um elemento fundamental no sistema de franchising, considerando que o principal ativo da rede franqueadora é sua marca. Na expansão internacional de redes de franquia, a reputação é um elemento importante (Fladmoe-Lindquist, 1996; Shane, 1996; Perrigot, López-Fernández \& Eroglu, 2013). Potenciais franqueados nos mercados de destino buscam franqueadores com boa reputação, para evitar comportamentos oportunistas e riscos. Estes potenciais franqueados no exterior podem acessar informações de fontes públicas e por meio de outros franqueados para consultar o histórico do franqueador em termos de qualidade, justiça, confiança e desempenho (Elango, 2007).

As redes de franquia recorrem a premiações e chancelas como mecanismos que as respaldem e promovam a aceitabilidade e reputação. No Brasil, um destes mecanismos é o Selo de Excelência em Franchising, promovido pela Associação Brasileira de Franchising desde 1990. Em 2018 a chancela estava em sua 28a edição.

"O Selo de Excelência em Franchising visa reconhecer a qualidade e excelência da empresa e sua atuação como franqueadora, e estimula a melhoria de seu desempenho, por meio da valorização das melhores práticas e do profissionalismo das redes que atuam no setor, através da avaliação criteriosa de seus franqueados." (ABF, 2016).

Portanto, a seguinte hipótese é apresentada:

H3: Franqueadores com a chancela "Selo de Excelência em Franchising" estão mais propensos a se internacionalizarem.

\section{IBV e Internacionalização de Franquias}

As organizações fazem parte de um ambiente institucional que exerce um certo grau de pressão e influência sobre elas. Ambientes institucionais têm como características a elaboração de regras e exigências a que as organizações devem obedecer se quiserem receber apoio e legitimidade (Garud et al., 2007). 
A visão baseada nas instituições (IBV, sigla em inglês) emergiu como uma forma de explicar as diferenças nas estratégias das firmas, e agregar às perspectivas de competitividade de mercado, ou visão da indústria, e visão baseada em recursos. Para a IBV além de se avaliar a indústria e os recursos, a firma precisa considerar influências mais amplas do seu ambiente institucional (Peng, 2002). Desta forma, a visão baseada em insituições integra a visão baseada na indústria com a visão baseada em recursos, uma combinação importante para endereçar questões relevantes com foco em "Negócios Internacionais" (Peng et al., 2008, p. 920).

As instituições interagem com as organizações e sinalizam as escolhas estratégicas aceitáveis em um determinado contexto. O ambiente institucional é formado por regras formais, que incluem regras políticas, decisões judiciais e contratos econômicos, e regras informais, como normas de comportamento socialmente aceitas, incorporadas na cultura e ideologia. Neste sentido, as instituições auxiliam a reduzir as incertezas para as organizações (Peng, 2002).

A visão institucional sobre o ponto de vista dos estudos de estratégia, concentra-se, portanto, na interação dinâmica entre instituições e organizações e considera escolhas estratégicas como o resultado de tal interação. Assim, as instituições têm impacto na estratégia e no desempenho da empresa.

\section{Pertencimento a Grupos}

A teoria institucional pressupõe que as empresas e gestores respondem à influência social e à pressão para a conformidade. A pressão social para se adequar às práticas e normas aceitas e legitimadas emergem tanto das empresas como dos seus ambientes intitucionais (Combs et al., 2009). Estas pressões e influências promovem a criação de estruturas e mecanismos que visam garantir legitimidade, reconhecimento, aceitabilidade e reputação (Scott in Smith \& Hitt, 2004).

Os processos institucionais afetam as decisões das redes de franquias e a teoria institucional ajuda a explicar a evolução do franchising. Os franqueadores que compartilham um ambiente institucional comum muitas vezes acabam tomando decisões semelhantes, decorrentes de processsos de isomorfismo. Isomorfismo pode ser definido como processo pelo qual as organizações imitam os caminhos percorridos por outras empresas, utilizando-as como modelos e forma de lidar com as incertezas do ambiente (Scott, 1995).

Portanto, redes de franquias que fazem parte de grupos empresariais tendem a seguir caminhos e estratégias semelhantes entre si. Quando redes de franquias pertencentes a um grupo já operam em mercados internacionais, podem facilitar que outras redes do mesmo grupo compartilhem a estratégia de internacionalização (Combs et al., 2009; Aliouche et al., 2015).

Além disso, no contexto de economias emergentes, empresas que operam em um ambiente institucional em desenvolvimento geralmente buscam diversificar seus produtos e indústrias para minimizar riscos. Elas podem alcançar a diversificação por meio de grupos empresariais. Ao fazer parte de um grupo, as firmas podem acessar recursos e capacidades de outras empresas. Os grupos também podem preencher vazios institucionais e garantir maior segurança às empresas (Gaur et al., 2013).

Portanto, a partir da visão institucional propõe-se a seguinte hipótese:

H4:. Franqueadores que pertencem a grupos estão mais propensos a se internacionalizarem

\section{METODOLOGIA}

A presente pesquisa analisa em que medida, (1) a experiência de monitoramento dos franqueados, (2) a dispersão geográfica no mercado doméstico, (3) reputação e (4) pertencimento a grupos - são ou não aspectos que impactam a internacionalização de redes de franquias, variáveis que podem explicar a ainda reduzida expansão das franquias brasileiras para o exterior.

Para o desenvolvimento do estudo, faz-se uso da Regressão Logística, método estatístico multivariado de dependência, que relaciona um conjunto de variáveis independentes, explicativas, com uma variável dependente, categórica - que assume valor 1 para o evento de ocorrência - a internacionalização de franquias, e zero para a não ocorrência, a não internacionalização. O Logit como função de regressão é um modelo estatístico capaz de investigar e predizer se um conjunto de variáveis explanatórias / preditoras influenciam ou não a ocorrência de um evento de interesse - a variável explicada, ou seja, se uma ou mais variáveis dependentes podem ser explicadas ou preditas por variáveis independentes (Gouvêa et al, 2012; Prearo et al, 2009). 
Fatores Relacionados a Internacionalização das Redes de Franquias Brasileiras: Dispersão Geográfica e Pertencimento a Grupos

\section{Coleta de Dados}

A amostra deste estudo é extraída de uma base de dados fornecida pela Associação Brasileira de Franchising (ABF) referente ao ano de 2017, que identifica 675 redes de franquias brasileiras. Esta amostra passou por um tratamento prévio dos dados, com a retirada de valores faltantes, tendo sido estabelecido critérios, filtros de identificação das redes de franquias selecionadas e suas respectivas unidades, entre os quais: redes de franquias cujo país de origem é identificado como Brasil e redes cujo número de unidades próprias e franqueadas é diferente de zero, chegando a uma amostra final de 420 redes de franquias. Destas 420 redes de franquia, 49 possuem operações em mercados estrangeiros e 371 não estão internacionalizadas; 55 redes de franquia pertencem a grupos e 365 não pertencem a grupos; 172 possuem Selo de Excelência ABF e 248 não possuem Selo de Excelência ABF. A média de unidades da amostra é 66 unidades, com desvio padrão de 99,5.

A ABF é a representante oficial do franchising brasileiro, organização sem fins lucrativos, criada em 1987, que possui mais de 1100 associados, entre franqueadores, franqueados e colaboradores. A Associação é membro-fundador do WFC (World Franchise Council), bem como da FIAF (Federação Ibero-Americana de Franquias). Integra ainda o quadro da IFA (International Franchise Association), e é membro correspondente da Federação Europeia de Franchising.

\section{Estabelecimento das Variáveis do Estudo}

\section{Variável Dependente}

A variável resposta também chamada de variável explicada ou dependente, tem caráter qualitativo, categorizado, e reflete uma escolha entre duas alternativas - redes de franquias internacionalizadas e redes de franquias não internacionalizadas. "A escolha é uma ou outra de duas alternativas disponíveis e mutuamente exclusivas" (Oliveira, 1997). Trata-se de uma variável dependente dicotômica, binária, ou variável dummy, com duas possibilidades de resposta - atribuindo-se valor 1 para o evento de interesse, de sucesso, e no caso deste estudo as franquias internacionalizadas, e o valor 0 para aquelas que não se internacionalizaram (Batistela et al, 2009; Oliveira, 1997).

Desta forma, a variável dependente dicotômica consiste em Redes de Franquias Internacionalizadas (valor 1) e Redes de Franquias Não Internacionalizadas (valor 0 ). Na base de 420 redes de franquias, verificou-se 49 internacionalizadas com valor 1 e 371 não internacionalizadas com valor 0.

Por redes de franquias internacionalizadas consideram-se redes com os seguintes arranjos organizacionais utilizados para conduzir seus negócios internacionais: 1 . Investimento Direto Estrangeiro, com a instalação de unidades próprias; 2. Instalação de unidades franqueadas; 3. Desenvolvedores de área; 4. Joint ventures e máster franchising (Elango \& Fried, 1997; Lee et al., 2012; Doherty \& Quinn, 1999; Jell-Ojobor \& Windsperger, 2013).

\section{Variáveis Independentes}

São quatro as variáveis independentes:

(1) Experiência de monitoramento - variável contínua, refere-se à proporção de unidades franqueadas em relação às unidades próprias multiplicado pelo número de anos que o franqueador atua no sistema de franchising.

(2) Dispersão geográfica nacional - variável contínua, tendo como proxy o número de municípios no mercado doméstico em que a rede está presente.

$\mathrm{Na}$ base de 420 redes de franquias, verificou-se redes com presença em no mínimo 1 município e no máximo em 364. A média de municípios da amostra é 37 municípios com desvio padrão de 53,8.

(3) Reputação - variável dicotômica, corresponde à atribuição de "Selo de Excelência" (valor 1) ou não atribuição (valor 0) - que representa reputação do mercado doméstico, idoneidade da empresa. Na base de 420 redes de franquias, verificou-se 172 com valor 1 e 248 com valor 0 .

(4) Pertencimento a Grupos - variável dicotômica, refere-se ao pertecencimento (valor 1) ou não pertencimento (valor 0 ) da rede de franquia a um grupo empresarial. Na base de 420 redes de franquias, verificou-se 55 com valor 1 e 365 com valor 0 .

\section{Variável de Controle}

Uma variável de controle distingue o grupo de franquias de acordo com o tamanho (unidades próprias e franqueadas).

O número mínimo de unidades próprias e franqueadas das redes de franquia que compõe a amostra é 2 unidades e o número máximo é 694 unidades. A média de unidades é 66 e o desvio padrão 99,5. 
RESULTADOS E DISCUSSÃO

Os resultados da regressão logística são reportados na Tabela 1. Para as variáveis independentes "experiência de monitoramento" e "dispersão geográfica nacional" e variável de controle "tamanho (unidades)" foram consideradas as transformações logarítmicas para linearização.

Tabela 1: Análise de regressão logística dos aspectos que influenciam a internacionalização de redes de franquia

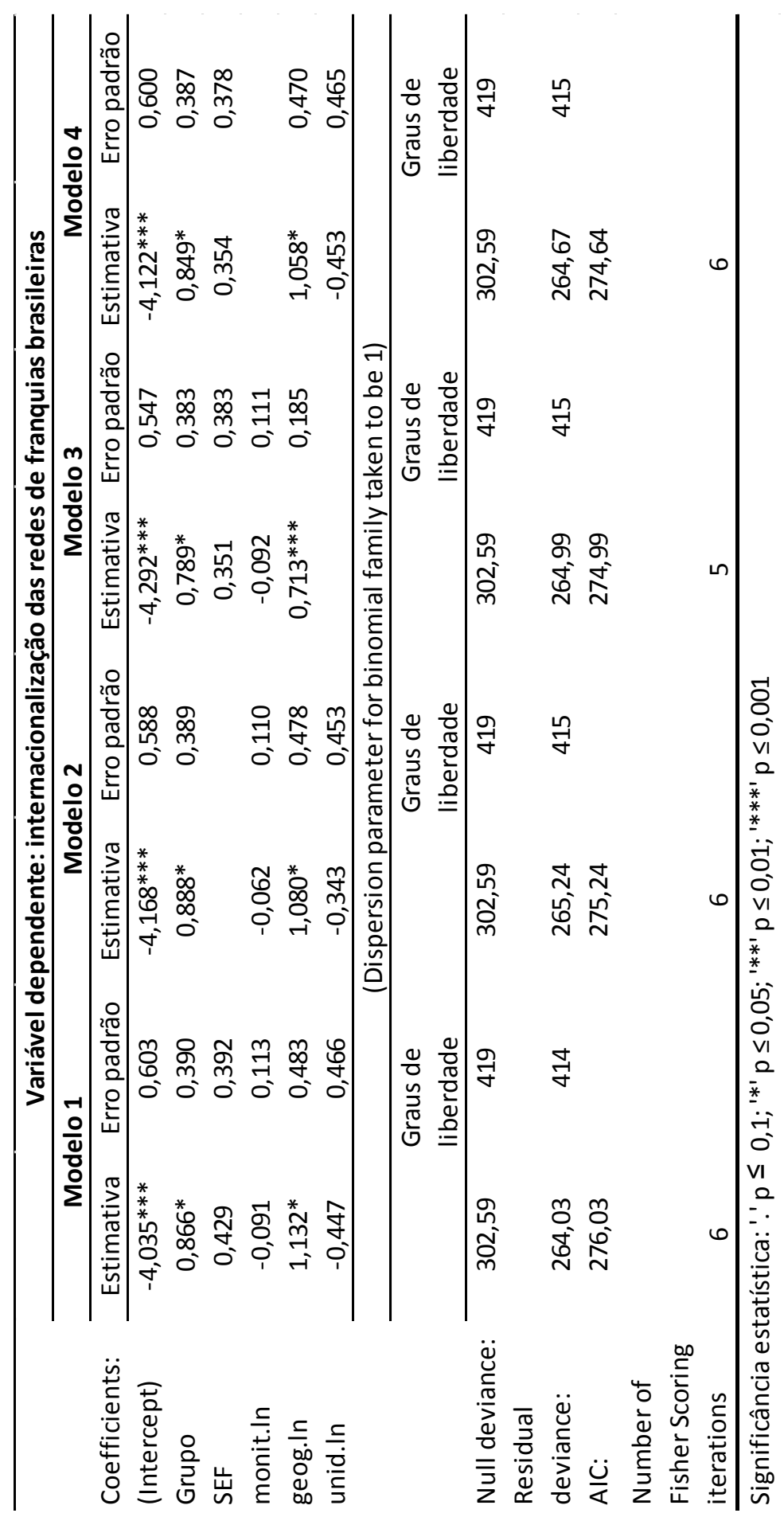

Fonte: Autores 
Fatores Relacionados a Internacionalização das Redes de Franquias Brasileiras: Dispersão Geográfica e Pertencimento a Grupos

O Modelo 1 apresenta os resultados da regressão logística considerando as quatro variáveis independentes e a variável de controle. Para testar a consistência dos resultados, o mesmo método foi aplicado a outros três modelos, que apresentam pequenas variações em relação ao Modelo 1 . O objetivo é confirmar a robustez do modelo base e verificar se seus resultados se mantém mesmo com a inclusão ou exclusão de algumas das variáveis.

No modelo 2 foi desconsiderada a variável independente Reputação (SEF); no Modelo 3 foi desconsiderada a variável de controle Tamanho (unid.In) e no Modelo 4 foi desconsiderada a variável independente Experiência de monitoramento (monit.In).

Em relação às variáveis caracterizadas como recursos da empresa (capacidade de monitoramento, dispersão geográfica e reputação), no Modelo 1 os resultados indicam que a dispersão geográfica nacional apresenta impacto positivo na propensão à internacionalização de redes de franquias a um nível de significância de 5\%.

Ou seja, a hipótese 2 foi confirmada e, portanto, franqueadores com maior dispersão geográfica no mercado doméstico estão mais propensos a se internacionalizarem.

Já a variável capacidade de monitoramento não apresentou resultado significativo neste estudo com amostra de redes de franquias brasileiras, portanto a hipótese 1 foi rejeitada.

A variável Reputação (SEF) se comportou da maneira esperada com coeficiente positivo, ou seja, é positivamente associada com a probabilidade de internacionalização, porém o resultado não foi estatísticamente significativo e a hipótese 3 foi rejeitada.

Em relação à variável caracterizada como recurso institucional (Pertencimento a grupos), os resultados sinalizam associação positiva entre Pertencimento a grupos e a probabilidade das redes de franquia internacionalizarem suas operações, a um nível de significância de 5\%. Portanto a hipótese 4, franqueadores que pertencem a grupos estão mais propensos a se internacionalizarem, foi confirmada pelos resultados.

Os modelos 2, 3 e 4 apresentam resultados semelhantes e confirmam as relações positivas e significativas entre as variáveis "dispersão geográfica nacional" e "pertencimento a grupos" com a probabilidade das redes de franquia se internacionalizarem. No modelo 3 , em que a variável de controle "tamanho (unidades)" foi desconsiderada, os resultados para a variável Dipersão geográfica nacional foram ainda mais significativos, indicando associação positiva a um nível de significância de $1 \%$.

Tabela 2: Resultados

\begin{tabular}{|l|l|}
\hline Hipótese & Resultado \\
\hline H1 - Experiência de monitoramento & Rejeitada \\
\hline H2 - Dispersão geográfica & Confirmada \\
\hline H3 - Reputação (Selo de Excelência) & Rejeitada \\
\hline H4 - Pertencimento a grupos & Confirmada \\
\hline
\end{tabular}

Fonte: Autores

A experiência de monitoramento não apresentou resultados significativos para a realidade das franqueadoras brasileiras, apesar de ter sido identificada como relevante em estudos anteriores com redes de franquia de outros países (Shane, 1996; Elango, 2007). A chancela Selo de Excelência em Franchising, apesar de que seus resultados tenham sido de acordo com o esperado, positivamente associado à propensão de internacionalização das franqueadoras, também não apresentou resultados estatísticamente significativos. Análises do impacto dessas duas variáveis para as franqueadoras brasileiras de segmentos de atuação distintos e em perspectiva longitudinal podem trazer resultados diferentes e, portanto, sugere-se como temas de estudos futuros. 
Aspectos institucionais como o pertencimento a grupos estimulam e fornecem certas capacidades, como troca de experiências e informações, que podem ser obtidas de outras formas, por exemplo a participação em Comunidades de Prática (CoP) e Redes de Prática (NoP). Comunidades de Práticas e Redes de Práticas são grupos criados para promover o compartilhamento de conhecimento entre pessoas e organizações (McFarland \& Gomez, 2013). Da mesma maneira, recursos da empresa como a dispersão geográfica nacional, que estimula o desenvolvimento de mecanismos de controle e monitoramento, podem compor uma estratégia de expansão internacional a longo prazo.

\section{CONCLUSÕES}

A partir de uma perspectiva multiteórica, este artigo buscou identificar fatores que explicam o baixo índice de internacionalização das redes de franquias brasileiras. Dos quatro aspectos avaliados, sendo três recursos da empresa (experiência de monitoramento, dispersão geográfica nacional e reputação) e um aspecto institucional (pertencimento a grupos), os resultados confirmaram as hipóteses relacionadas à dispersão geográfica nacional e ao pertencimento a grupos.

Foi possível observar a partir dos resultados que a dispersão geográfica nacional das redes de franquias brasileiras, medida pelo número de municípios no mercado doméstico em que a rede está presente, apresenta influência positiva na probabilidade da franqueadora internacionalizar suas operações. Ou seja, uma maior dispersão geográfica exige e estimula o desenvolvimento de capacidades operacionais e de gestão, como controle e logística, que podem tornar a rede franqueadora mais preparada e apta a operar em mercados estrangeiros. As redes de franquia que compõe a amostra analisada estão presentes em média em 37 municípios do Brasil. As redes de franquia da amostra que são internacionalizadas (49 redes) estão presentes em 82 municípios, em média. Já as redes da amostra que não são internacionalizadas (371 redes) estão presentes em 31 municípios em média. Isto sinaliza uma baixa

\section{REFERÊNCIAS}

ABF (2016). Números do Franchising. Associação Brasileira de Franchising. Disponível em https://www.abf.com.br/numeros-do-franchising/

Aliouche, E. H., Fernandez, D. B., Chanut, O. \& Gharbi, N. (2015). Institutional Environments And The dispersão geográfica, o que pode contribuir para o baixo índice de internacionalização das redes de franquias brasileiras. $O$ fato delas já terem crescido no Brasil, e alcançado uma forte penetração em vários municípios contribui para elas terem recursos para internacionalizar.

O pertencimento das redes franqueadoras a grupos empresariais também apresentou associação positiva com a probabilidade de internacionalização das redes de franquias brasileiras. Aquelas redes que pertencem a grupos que já operam em mercados internacionais podem ser beneficiadas pelo compartilhamento de informações e experiências derivadas da estratégia de internacionalização. Este fator facilita o crescimento e a troca dentro dos grupos, o que facilita a busca por oportunidades no exterior.

Da amostra de redes de franquias analisada, apenas $13,1 \%$ pertence a grupos empresariais. Como os resultados indicam que o pertencimento a grupos está positivamente relacionado à internacionalização, o pequeno número de redes que compartilham experiências e informações em grupos pode contribuir para o baixo índice de internacionalização.

Este estudo contribui do ponto de vista teórico ao aplicar duas perspectivas teóricas complementares, visão baseada em recursos e visão institucional, à análise de um fenômeno de franchising internacional (Comb et al., 2009).

$\mathrm{O}$ artigo também atende às sugestões de estudos sobre franchising internacional em mercados emergentes (Dant, Grünhagen \& Windsperger, 2014). Como contribuições gerenciais, o entendimento dos aspectos que aumentam a probabilidade de uma rede de franquia brasileira se internacionalizar pode contribuir para que os gestores de redes franqueadoras desenvolvam capacidades ou se adaptem a certas condições visando a expansão internacional. Ou seja, esta pesquisa é relevante para o setor de franchising pois fornece subsídios para a implantação das estratégias de internacionalização de franquias.

Internationalization Of Franchise Chains: The Contrasting Cases Of Three North African Countries. The Journal of Applied Business Research, 31(2), 1-33.

Alon, I. (2000). Organizational Determinants of Master International Franchising. Journal of Business and Entrepreneurship, 12, 1-18. 
Fatores Relacionados a Internacionalização das Redes de Franquias Brasileiras: Dispersão Geográfica e Pertencimento a Grupos

Alon, I., Nib, L., \& Wangc, Y. (2012). Examining the determinants of hotel chain expansion through international franchising. International Journal of Hospitality Management, 31 (2), 379-386.

Aydin, N., \& Kacker, M. (1990). International Outlook of US-based Franchisers. International Marketing Review, (7)2, 43-53.

Barney, J. (1991). Firm Resources and Sustained Competitive Advantage. Journal of Management, 17(1), 99-120.

Batistela, G. C.; Rodrigues, S. A.; Bonomi, J.T.C.M. (2009). Estudo sobre a evasão escolar usando regressão logística: análise dos alunos do curso de administração da fundação educacional de Ituverava. Tékhne $\varepsilon$ Lógos, Botucatu, 1 (1), 54-67.

Bianchi, C., \& Arnold, S. J. (2004). An institutional perspective on retail internationalization success: home depot in Chile. The International Review of Retail, Distribution \& Consumer Research, 14 (2), 149169.

Bianchi, C. (2009). Retail internationalisation from emerging markets: case study evidence from Chile. International Marketing Review, 26(2), 221 - 243.

Castrogiovanni, G. J., Combs, J. G., \& Justis. R. T. (2006). Resource Scarcity and Agency Theory Predictions Concerning the Continued Use of Franchising in Multi-outlet Networks. Journal of Small Business Management, 44(1), 27-44.

Cavusgil, S. T., Knight, G. A., \& Riesenberger, J. R. (2010). Negócios internacionais: estratégia, gestão e novas realidades. São Paulo: Pearson.

Combs, J. G., Michael, S. C., \& Castrogiovanni, G. J. (2009). Institutional Influences on the Choice of Organizational Form: The Case of Franchising. Journal of Management, 35 (5), 1268-1290.

Dant, R. P., Grunhagen, M., \& Windsperger, J. (2014) Franchising Research Frontiers for the Twenty-First Century. Journal of Retailing, 87 (3), 253-268.

Doherty, A. M. (2007). The internationalization of retailing. Factors influencing the choice of franchising as a market entry strategy. International Journal of Service Industry Management, 18 (2), 184-205.

Elango, B. (2007). Are Franchisors with International Operations Different from Those Who Are Domestic Market Oriented? Journal of Small Business Management, 45 (2), 179-193.
Eroglu, S. (1992). The Internationalization Process of Franchise Systems: A Conceptual Model. International Marketing Review, 9(5).

International Franchise Association. https://www.franchise.org/international-franchiseopportunities

Fladmoe-Lindquist, K. (1996). International franchising: Capabilities and development. Journal of Business Venturing, 11 (5), 419-438.

Fundação Don Cabral. Ranking FDC das Multinacionais Brasileiras (2016). https://www.fdc.org.br/professoresepesquisa/nucle os/Documents/negocios internacionais/2016/Ranki ng FDC Multinacionais Brasileiras 2016.pdf

Acesso em 061217.

Garud R., Hardy, C., \& Maguire S. (2007). Institutional Entrepreneurship as embedded agency: an introduction to the special issue. Organization Studies, 28 (7), p. 957-969.

Gaur, A. S., Kumar, V. \& Singh, D. (2014). Institutions, resources, and internationalization of emerging economy firms. Journal of World Business, 49 (1), 12-20.

Gouvêa, M. A.; Prearo, L. C.; Romeiro, M. C. Avaliação da adequação de aplicação de técnicas multivariadas em estudos do comportamento do consumidor em teses e dissertações de duas instituições de ensino superior. R.Adm., 47 (2), 338355.

Huszagh, S., Huszagh, F., \& Mclntyre, F. (1992). International franchising in the context of competitive strategy and the theory of the firm. International Marketing Review, 9 (5), 5-18.

Khanna T., \& Palepu, K. (2010). Winning in Emerging Markets: A Road Map for Strategy and Execution. Boston: Harvard Business School Press.

Kedia, B.L., Ackerman, D. J., Bush, D. E. \& Justis, R. T. (1994). Determinants of Internationalization of Franchise Operations by US Franchisors. International Marketing Review, 11 (4), 56-68.

Lockett, A., Thompson, S., \& Morgenstern, U. (2009) The development of the resource-based view of the firm: A critical appraisal. International Journal of Management Reviews, 11 (1), 9-28.

Moraes, W. F. A., Oliveira, B. R. B., \& Kovacs, E. P. (2006). Teorias de internacionalização e aplicação em países emergentes: uma análise crítica. Internext - 
Revista Eletrônica de Negócios Internacionais, 1 (1), 203-220.

Madanoglu, M., Alon, I., \& Shoham, A. (2017). Push and pull factors in international franchising. International Marketing Review, 34 (1), 29-45.

McFarland, D. A., \& Gomez, C. J. (2013). Organizational Analysis.

Oliveira, M. M. Econometria. Alfragide, Portugal: McGraw-Hill, 1997.

Osland, G. E., Taylor, C. R., \& Zou, S. (2001). Selecting international modes of entry and expansion. Marketing Intelligence \& Planning, 19(3), $153-161$.

Pedro, M., Filipe, J., \& Reis, E. (2008). Factores Determinantes da Internacionalização das Redes de Franchising Ibéricas. Economia Global \& Gestão. XIII. 86-84.

Peng, M. W. (2001). The resource-based view and international business. Journal of Management, 27 (6), 803-829.

Peng, M. W. (2002). Towards an Institution-Based View of Business Strategy. Asia Pacific Journal of Management, 19 (2-3), 251-267.

Peng, M. W., Wang, D. Y. L., \& Jiang, Y. (2008). An institution-based view of international business strategy: a focus on emerging economies. Journal of International Business Studies, 39(5), p.920-936.

Perrigot, R., López-Fernández, B., \& Eroglu, S. (2013). Intangible Resources and Plural Form as Drivers of Franchise Internationalization: Examination within a Two-Country Perspective. Journal of Small Business Management, (4), 557-577.
Porter, M. E. (1980) Competitive strategy: techniques for analysing industries and competitors. New York: Free Press.

Porter, M. E. (1985). Competitive advantage:creating and sustaining competitive performance. New York: Free Press.

Prearo, L. C, Gouvêa, M. A., \& Monari, C. (2009). Avaliação do emprego da técnica de análise de regressão logística em teses e dissertações de algumas instituições de ensino superior. Semina: Ciências Sociais e Humanas, 30(2), p. 123-140.

Quinn, B., \& Alexander, N. (2002). International retail franchising: a conceptual framework. International Journal of Retail \& Distribution Management, 30(5), 264 $-276$.

Rocha, T.V., Spers, E. E., Borini, F. M., Bretas, V. P., Melo, P. L. R., Ogasavara, M. H., Khauaja, D. M. R., \& Camargo, M. A. A. (2016). Global mindset na internacionalização das franquias brasileiras. 4a ed. v. 1. São Paulo: ESPM.

Sashi, C.M., \& Karuppur, D. P. (2002). Franchising in global markets: towards a conceptual framework. International Marketing Review, 19(5), 499 - 524.

Scott, W. R. (1995). Institutions and organizations. Sage Publications.

Scott, W. R. Institutional Theory: Contributing to a Theoretical Research Program in Smith, K. G., \& Hitt, M. A. (2005). Great Minds in Management: The Process of Theory Development. Oxford UK: Oxford University Press.

Welsh, D. H. B., Alon, I., \& Falbe, C. M. (2006). An Examination of International Retail Franchising in Emerging Markets. Journal of Small Business Management, 44 (1), 130- 149.

\section{SOBRE OS AUTORES}

- Vanessa Pilla Bretas - Doutoranda em Administração na Escola Superior de Propaganda e Marketing - ESPM, São Paulo, (Brasil). E-mail: vpbretas@gmail.com Orcid.id: https://orcid.org/0000-0003-4894-8557

- Jefferson Ricardo Galetti - Doutor em Economia da Indústria e da Tecnologia pela Universidade Federal do Rio de Janeiro - UFRJ, Rio de Janeiro, (Brasil). E-mail: jeffersongaletti@gmail.com Orcid.id: https://orcid.org/0000-0001-5582-1875

- Thelma Valéria Rocha - Doutora em Administração de Empresas pela Faculdade de Economia, Administração, Contabilidade e Atuária da Universidade de São Paulo -FEA/USP, São Paulo, (Brasil). E-mail: tvrocha@espm.br Orcid.id: https://orcid.org/0000-0002-3825-4343 
Fatores Relacionados a Internacionalização das Redes de Franquias Brasileiras: Dispersão Geográfica e Pertencimento a Grupos

\title{
Organizational Socialization of Expatriates
}

\author{
Vanessa Pilla Bretas, Jefferson Ricardo Galetti, Thelma Valéria Rocha \\ Escola Superior de Propaganda e Marketing - ESPM, São Paulo, (Brazil) \\ Universidade Federal do Rio de Janeiro, Rio de Janeiro, (Brazil)
}

ARTICLE DETAILS
Invited paper:
Received: 16 august 2018
Accepted: 23 december 2018
Available online January: 01th 2019
Scientifc Editor
Ilan Avrichir

\section{Keywords:}

International Franchising

Franchising

Internationalization

Geographic dispersion

\begin{abstract}
This paper investigates the factors that explain the low internationalization rate of Brazilian franchise chains. We seek to identify whether the company's resources, such as monitoring experience, geographic dispersion in the domestic market and reputation, and institutional aspects, such as business groups affiliation, influence the probability of Brazilian franchise networks expand overseas. Using data provided by the Brazilian Franchising Association (ABF), the relation between these four aspects and the chances of internationalization are investigated through a logistic regression for a base of 420 Brazilian franchise chains, 49 internationalized and 371 not internationalized. The results confirmed the hypotheses related to the national geographic dispersion, franchisors with greater geographic dispersion in the domestic market are more likely to internationalize, and business group affiliation, franchisors affiliated to groups are more likely to internationalize. This study innovates by quantitatively proving that national geographic dispersion and business group affiliation are factors that determine internationalization of emerging market franchise chains, such as Brazil.
\end{abstract}

(C) 2018 Internext | ESPM. All rights reserved!

\section{Para citar este artigo:}

Bretas, V., Galetti, J., \& Rocha, T. (2019). Fatores Relacionados a Internacionalização das Redes de Franquias Brasileiras: Dispersão Geográfica e Pertencimento a Grupos. Revista Eletrônica de Negócios Internacionais, 14(1), 01-13. doi:https://doi.org/10.18568/internext.v14i1.495 